\title{
Preparation of Cellulose Nanofibril/Regenerated Silk Fibroin Composite Fibers
}

\author{
Ji Hye Lee ${ }^{1}$, Chang Hyun Bae ${ }^{1}$, Byung-Dae Park ${ }^{2}$, and In Chul Um ${ }^{1 *}$ \\ ${ }^{I}$ Department of Bio-fibers and Materials Science, Kyungpook National University, Daegu 702-701, Republic of Korea \\ ${ }^{2}$ Department of Wood Science and Technology, Kyungpook National University, Daegu, 702-701, Republic of Korea
}

\section{Abstract}

Wet-spun silk fibers have attracted the attention of many researchers because of 1) the unique properties of silk as a biomaterial, including good biocompatibility and cyto-compatability and 2) the various methods available to control the structure and properties of the fiber. Cellulose nanofibrils (CNFs) have typically been used as a reinforcing material for natural and synthetic polymers. In this study, CNF-embedded silk fibroin (SF) nanocomposite fibers were prepared for the first time. The effects of CNF content on the rheology of the dope solution and the characteristics of wet-spun CNF/SF composite fibers were also examined. A $5 \%$ SF formic acid solution that contained no CNFs showed nearly Newtonian fluid behavior, with slight shear thinning. However, after the addition of $1 \%$ CNFs, the viscosity of the dope solution increased significantly, and apparent shear thinning was observed. The maximum draw ratio of the CNF/SF composite fibers decreased as the CNF content increased. Interestingly, the crystallinity index for the silk in the CNF/SF fibers was sequentially reduced as the CNF content was increased. This phenomenon may be due to the fact that the CNFs prevent $\beta$-sheet crystallization of the SF by elimination of formic acid from the dope solution during the coagulation process. The CNF/SF composite fibers displayed a relatively smooth surface with stripes, at low magnification $(x 500)$. However, a rugged nanoscale surface was observed at high magnification (x10,000), and the surface roughness increased with the CNF content.

Received : 10 May2013

Accepted : 20 May 2013

Keywords:

Cellulose nanofibrils, Wet spun silk fiber, Composite,

Rheology,

Crystallinity index, Post drawing

\section{Introduction}

It has been previously reported that regenerated silk fibroin (SF) is highly compatible with blood (Sakabe et al., 1989; Um et al., 2002), has excellent cyto-compatibility (Minoura et al., 1995), and displays low inflammatory reactions in the body (Meinel et al., 2005). Additionally, regenerated SF fibers have several advantages: 1) the fiber morphology (thickness and shape) can be controlled through a variety of methods, 2) new materials, including enzymes and drugs, can be added to the regenerated silk fiber, and 3) the structure and properties can be varied depending on the application. Therefore, regenerated silk fiber has attracted the attention of researchers because of its extensive utilization in biomedical applications, including surgical sutures, tissue engineering scaffolds, drug delivery systems, and wound dressings.

\section{*Corresponding Author:}

In Chul Um

Department of Bio-fibers and Materials Science, Kyungpook National University, Daegu 702-701, Republic of Korea Tel: +82-53-950-7757, FAX: +82-53-950-6744

E-mail: icum@knu.ac.kr 
Several studies have been conducted on the wet spinning of silk, and many researchers have tried to find a new and effective solvent/coagulant system to fabricate regenerated silk fibers with desirable mechanical properties (Trabbic and Yager, 1998; Liivak et al., 1998; Um et al., 2004; Phillips et al., 2005; Ki et al., 2007; Ko and Um, 2009; Yan et al., 2010; Yoo et al., 2010; Cho et al., 2012). However, the mechanical properties of wet-spun silk filaments were not suitable for commercialization in a variety of contexts, including medical and textile applications.

Cellulose nanofibrils (CNFs) are typically obtained from cellulose by various means, including chemical and physical treatments (Klemm et al., 2011), and subsequently used in the preparation of composite nanocomposite films to improve the mechanical properties of the matrix material. For example, CNFs are used as reinforcing agents to prepare nanocomposites, including cellulose/polyurethane (Aksoy et al., 2007), cellulose/polyvinyl alcohol (Bhatnagar and Sain, 2005), cellulose/starch (Dufresne and Vignon, 1998), cellulose/poly(ethyleneoxide) (Brown and Laborie, 2007), and cellulose/chitosan (Nordqvist et al., 2007).

Recently, in relation to regenerated silk, the preparation of the nanocomposite material using nanosized cellulose has been studied by a plethora of researchers. Huang et al. (2011) prepared electrospun SF nanofiber mats reinforced by cellulose nanowhiskers (CNWs). They reported that the tensile strength and Young's modulus of the reinforced SF nanofiber mats were almost twice those of unreinforced SF mats when the $\mathrm{CNW}$ content was $2 \%(\mathrm{w} / \mathrm{w})$. Additionally, Park et al. (2012) fabricated nanofibrous bacterial cellulose nanocrystal (BCN)-embedded SF by using an electrospinning technique and found that Young's modulus of the SF nanofibers could be increased significantly by the addition of the BCNs. Li et al. (2012) prepared SF/ poly(ethylene glycol)/CNW composite films and reported that the mechanical properties of the SF film could be improved significantly by incorporating CNWs.

Although several researchers have reported nanocellulose-based silk composite films and electrospun fibers, an experiment concerning wet-spun nanocellulose/SF composites has not been performed until now. In this study, CNFs were added to $\mathrm{SF}$ to prepare $\mathrm{CNF} / \mathrm{SF}$ fibers using a wet-spinning technique, and the effect of the CNF content on the rheological properties, wet-spinnability, molecular conformation of the fiber, and fiber morphology was examined.

\section{Materials and Methods}

\section{Isolation of CNFs}

CNFs were isolated from cellulose pulp according to the modified TEMPO oxidation method (Saito et al. 2004). In short, the kraft pulp sample ( $2 \mathrm{~g}$ of cellulose) was suspended in water $(150 \mathrm{~mL})$ containing TEMPO $(0.025 \mathrm{~g})$ and sodium bromide $(0.25 \mathrm{~g})$. The TEMPO-mediated oxidation of the cellulose slurry was initiated by adding $5 \mathrm{mmol}$ of $13 \% \mathrm{NaClO}$ for every gram of cellulose and carried out at room temperature under gentle agitation. The $\mathrm{pH}$ was maintained at 10.5 by adding 0.5 $\mathrm{M} \mathrm{NaOH}$. When a decrease in $\mathrm{pH}$ was no longer observed, the reaction was considered complete, and the $\mathrm{pH}$ was then adjusted to 7 by adding $0.5 \mathrm{M} \mathrm{HCl}$. The TEMPO-oxidized product was filtered, thoroughly washed with water, and physically fibrillated by ultrasonication in a sonicator (Sonosmasher, Jeio Tech, Korea) for 20 minute. The suspension was then centrifuged at $5000 \mathrm{rpm}$ two or three times for 30 minute. The supernatant, which was in fact a suspension containing the CNFs, was decanted and collected. The yield of the CNFs was calculated as a percentage of the initial weight of the CNF suspension after drying in an oven at $105^{\circ} \mathrm{C}$. The final concentration of the CNFs was adjusted to $0.1 \%$ by weight/volume.

\section{Preparation of regenerated SF}

The method for preparing the regenerated SF was introduced in a previous study (Yoo et al., 2010). Bombyx mori silk cocoons were degummed with an aqueous solution containing $0.3 \%$ sodium oleate (o.w.f.) and $0.2 \%$ sodium carbonate (o.w.f.), which was boiled for 1 hour, and rinsed thoroughly with distilled water to obtain the SF. The SF was then dissolved in a solution of $\mathrm{CaCl}_{2} / \mathrm{H}_{2} \mathrm{O} / \mathrm{EtOH}$ (molar ratio=1/8/2) for 3 minute, with a liquor ratio of 1:20. The aqueous SF solution was obtained through dialysis of the SF solutions in a cellulose tube (molecular cutoff $=12,000-14,000 \mathrm{Da}$ ) against circulating purified water for 4 days at room temperature. The purified water was obtained by a Water Purification System (RO50, Hana Science, South Korea) 
with a reverse osmosis membrane. The SF solutions were then dried to obtain the regenerated SF polymers.

\section{Preparation of CNF/SF composite fibers}

A 5\% (w/w) regenerated SF formic acid solution was prepared by dissolving the regenerated SF in $98 \%$ formic acid. Approximately 1-5 wt\% (against the dry mass of regenerated $\mathrm{SF}$ ) of CNFs was added to the 5\% SF formic acid solution to prepare $\mathrm{CNF} / \mathrm{SF}$ dope solutions. Prior to wet spinning, the $\mathrm{CNF} / \mathrm{SF}$ dope solutions were filtered twice through nonwoven fabric to remove any insoluble particles. The CNF/SF composite fibers were spun using a syringe and syringe pump by extruding the dope solution through a 26-gauge needle (inner diameter $=0.241$, with a needle length of 0.5 inch) into methanol, which acts as a coagulation bath. The flow rate of the fiber extrusion was controlled at $20 \mathrm{~mL} / \mathrm{h}$. The as-spun $\mathrm{SF}$ filaments were left to stand in the coagulant overnight to allow the solvent (formic acid) to diffuse out completely from the filament prior to post-drawing and drying.

\section{Rheological measurement}

The CNF/SF dope solutions for wet spinning were used for the rheological measurements. The complex viscosity of the solutions was measured by rheometry (MARS III, Hakke, Germany), using a cone and plate geometry at $25^{\circ} \mathrm{C}$. An oscillation test was performed with a strain of $0.01 \%$. The radius and angle of the cone were $60 \mathrm{~mm}$ and $1^{\circ}$, respectively.

\section{Maximum draw ratio measurement}

The maximum draw ratio was calculated from the ratio of the maximum drawn length of the fiber and the length of the as-spun fiber in the wet state. The fiber length measurements were performed on 20 different parts of the filament, and the maximum draw ratio was determined by averaging the 20 results (Cho et al. 2012).

\section{FTIR measurement and determination of crystallinity index}

Fourier transform infrared (FTIR) (Nicolet 380, Thermo
Fisher Scientific, USA) spectra were obtained using the ATR method. The crystallinity index was calculated as the intensity ratio of the peaks at $1260 \mathrm{~cm}^{-1}$ and $1235 \mathrm{~cm}^{-1}$ from the FTIR spectrum, using the following equation (Bhat and Nadiger, 1980):

Crystallinity index $(\%)=\frac{A_{1260 \mathrm{~cm}^{-1}}}{A_{1235 \mathrm{~cm}^{-1}}} \times 100$

$\mathrm{A}_{1235 \mathrm{~cm}}{ }^{-1}:$ Absorbance at $1235 \mathrm{~cm}^{-1}$

$\mathrm{A}_{1260 \mathrm{~cm}}{ }^{-1}$ : Absorbance at $1260 \mathrm{~cm}^{-1}$

To obtain the average and variation of the crystallinity index, FTIR measurements were performed on five different portions of the samples.

\section{SEM observation}

The morphology of the CNF/SF composite fibers was observed by field-emission SEM observation (FE-SEM, S-4300, Hitachi, Japan) after coating them with gold.

\section{Results and Discussion}

\section{Rheological properties and wet spinnability of dope solution}

The rheological properties of the dope solution were examined since they strongly affect the fiber formation performance when using wet-spinning or electrospinning techniques (Cho et al., 2012; Ko et al., 2013). In the wet spinning of the regenerated silk, at least a viscosity of 70 $\mathrm{mPa} \cdot \mathrm{s}$ was needed for fiber formation in the methanol coagulant (Kim and Um, 2011; Chung and Um, 2011). This result indicates that a certain level of viscosity is necessary to fabricate the wet-spun regenerated silk fibers.

To understand the change in the rheological properties of the regenerated silk/formic acid solution after adding the CNFs, the complex viscosity of the dope solution was measured; the results are shown in (Fig. 1). The 5\% regenerated SF solution without CNFs showed almost Newtonian fluid behavior, although slight shear thinning was observed. This is consistent with the shear viscosity 


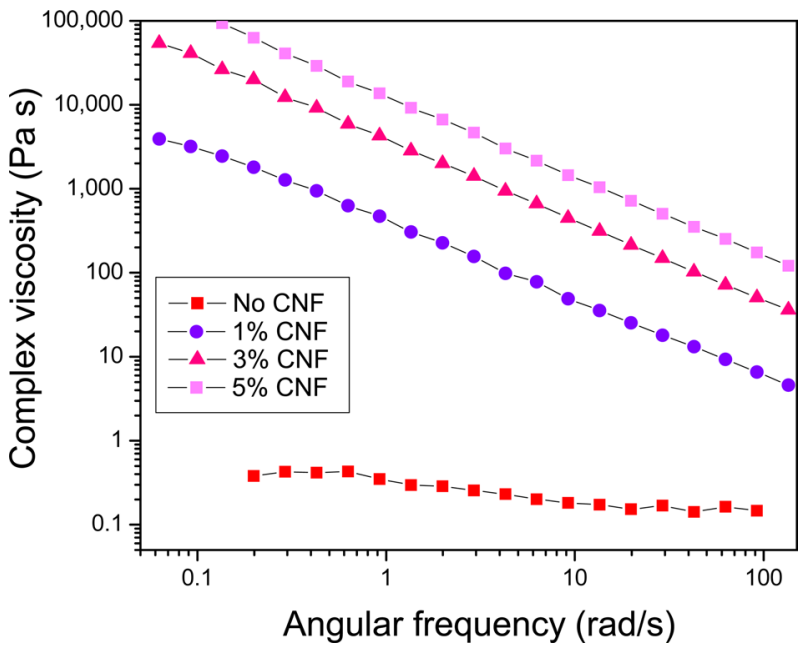

Fig. 1. Compolex viscosity of $\mathrm{CNF} /$ regenerated $\mathrm{SF}$ dope solution with various $\mathrm{CNF}$ contents.

results of SF formic acid solutions (Ko et al., 2013; Kim and Um, 2011; Chung and Um, 2011). When 1\% (w/w) CNFs was added to the $5 \%$ regenerated silk solution, the complex viscosity of the solution increased significantly and shear thinning was quite apparent. As the added amount of CNFs increased, the shear viscosity also increased, while the shear thinning behavior remained unchanged. The notable viscosity increase of the $1 \% \mathrm{CNF} /$ regenerated SF solution could be due to the fact that the CNFs exist as a suspension and aggregate in solution.

\section{Post-drawing performance of wet-spun filament}

Fiber orientation is a very important characteristic since it determines the mechanical properties of the fiber. In particular, the breaking strength of the fiber tends to be higher as the fiber orientation of wet-spun filaments increases. Um et al. (2004) reported that the breaking strength of the wet-spun regenerated silk fiber could be increased significantly by drawing the fiber with a draw ratio of 4 . In synthetic fiber research, many experiments have been conducted to increase the fiber drawing or takeup speed. Based on these background studies, the degree to which a fiber can be drawn is concluded to be important, and the maximum draw ratio (the ratio of the length of drawn fiber to the length of as spun fiber) has been used as a barometer to evaluate the wet spinnability of a given wet-

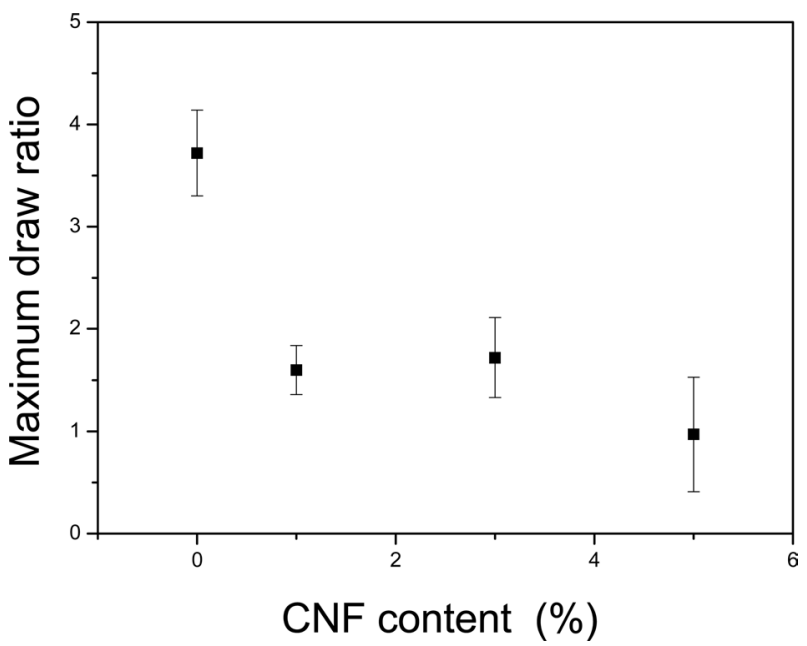

Fig. 2. Maximum draw ratio of $\mathrm{CNF} /$ regenerated SF filament with various $\mathrm{CNF}$ contents.

spun fiber (Yoo et al., 2010; Cho et al., 2010 and 2012).

(Fig. 2) shows the maximum draw ratio of $\mathrm{CNF} /$ regenerated silk filaments with different CNF contents. Without CNFs, the regenerated silk filament showed a maximum draw ratio of 3.7. However, as more CNFs were added to the silk, the maximum draw ratio decreased. This result indicated that the post drawing ability deteriorates with increasing CNF content, probably because of the inhomogeneous dope solution. Thus, the regenerated silk solution without CNFs is homogeneous and transparent. However, as the CNFs are added to the silk solution, the dope solution becomes turbid, with a higher degree of CNF and silk aggregation. In other words, the dope solution becomes inhomogeneous upon the addition of CNFs, and this solution with cellulose aggregates can prevent homogenous fiber spinning, resulting in beads and beaded fibers. The beads and beaded fibers can subsequently restrict post-drawing of the as-spun $\mathrm{CNF} /$ silk fibers, resulting in a decrease in the maximum draw ratio.

\section{Molecular conformation of wet-spun filaments}

The molecular conformation of the silk was examined since it strongly affects the chemical and physical properties of the silk polymers. $\beta$-sheet conformations help in the formation of crystalline regions of the silk, whereas random-coil conformations contribute to the formation of the amorphous region. Therefore, when the amount of 


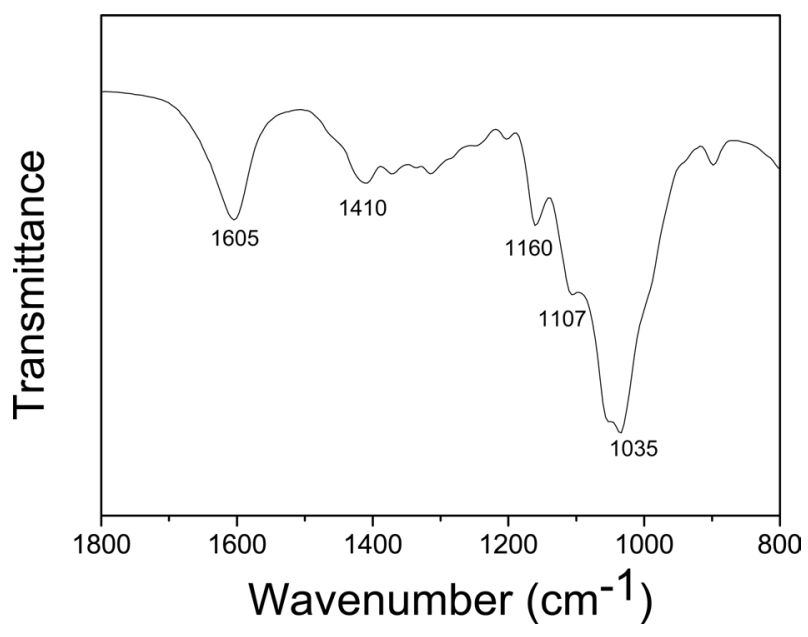

Fig. 3. FTIR spectrum of the CNFs used in this study.

$\beta$-sheets increases, the silk fiber becomes stronger, less water-soluble, and less degradable. FTIR analysis was used to examine the molecular conformation of the silk since different conformations yield absorption peaks at different positions in Amides I, II, and III.

(Fig. 3) displays the FTIR spectrum of the CNFs used in this study. Strong absorption peaks appeared at $1035 \mathrm{~cm}^{-1}$, which corresponded to stretching of the $\mathrm{C}-\mathrm{O}$ and $\mathrm{C}-\mathrm{C}$ bonds of cellulose, and at $1159 \mathrm{~cm}^{-1}$, which could be attributed to the antisymmetric C-O-C bridge stretching (Um et al., 2012).

(Fig. 4) shows the FTIR spectra of wet-spun CNF/ regenerated SF composite fibers with varying $\mathrm{CNF}$ contents. All the composite fibers showed strong absorption peaks at $1624 \mathrm{~cm}^{-1}$ and $1515 \mathrm{~cm}^{-1}$ and a shoulder peak at $1260 \mathrm{~cm}^{-1}$. These peaks are attributed to the $\beta$-sheet conformation, indicating that $\beta$-sheet crystallites exist in all the $\mathrm{CNF} / \mathrm{SF}$ composite fibers. It has been reported that $\beta$-sheet crystallization in the SF occurs when formic acid is removed (Um et al., 2003 and 2004). Interestingly, the shoulder peak at $1260 \mathrm{~cm}^{-1}$ increased slightly as the CNF content increased.

The crystallinity index of the silk proposed by Baht and Nadiger (1980) has been used to evaluate the crystallinity of the SF since it allows for a quantitative evaluation of this characteristic (Um et al., 2001; Kim et al., 2013).

Therefore, detailed calculations performed to determine the crystallinity index; the results are shown in (Fig. 5). The regenerated SF filament without CNFs exhibited a

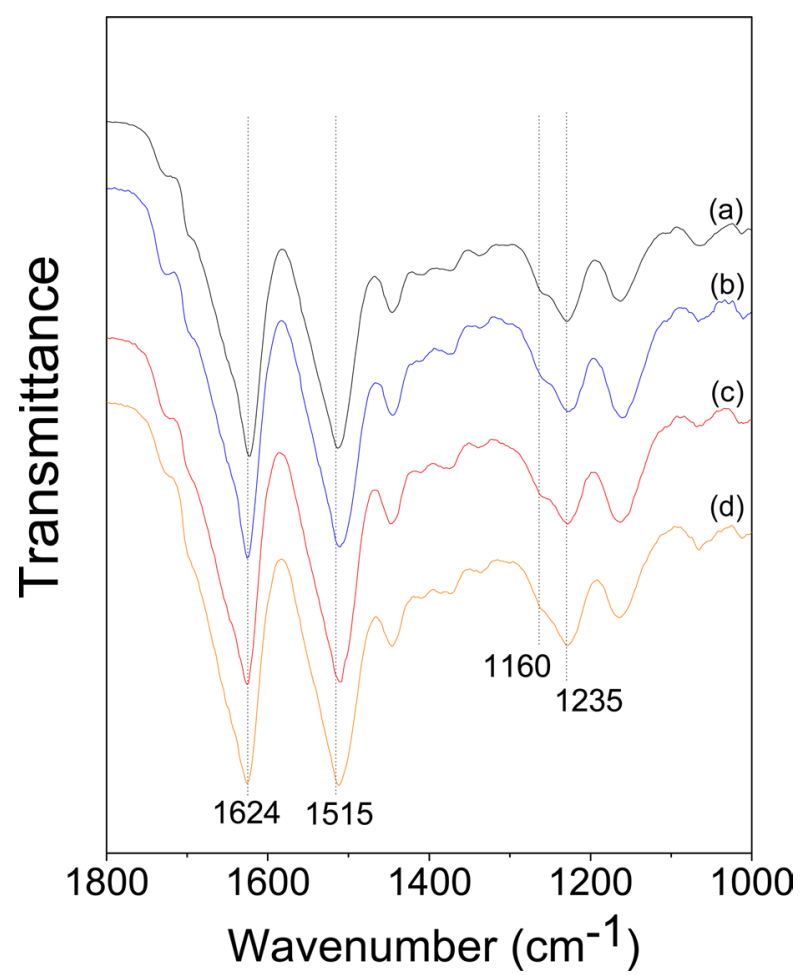

Fig. 4. FTIR spectra of CNF/regenerated SF filaments with various CNF contents: (a) $0 \%$, (b) $1 \%$, (c) $3 \%$, and (d) $5 \%$.

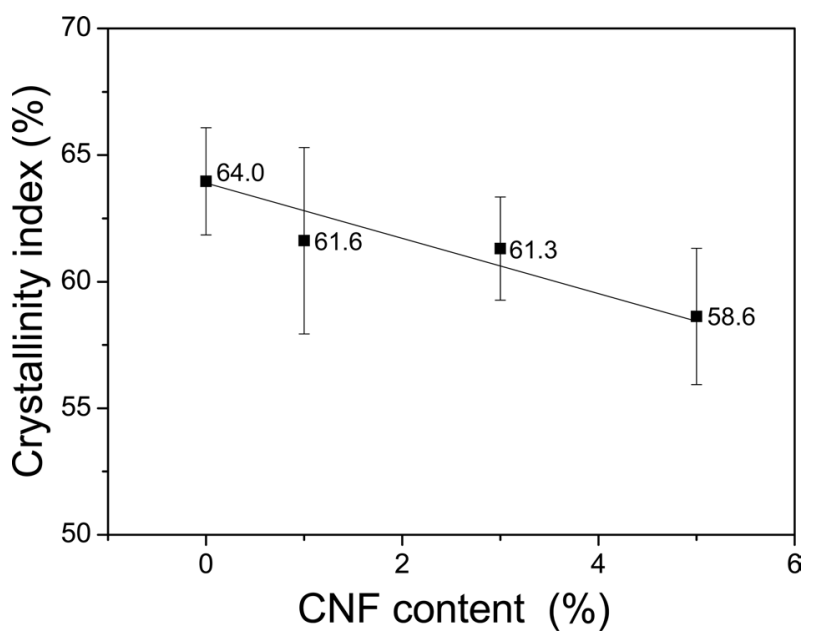

Fig. 5. Crystallinity index of $\mathrm{CNF} /$ regenerated SF filaments with various CNF contents.

crystallinity index of $64.0 \%$. However, the crystallinity index decreased as the amount of CNFs increased, with the $5 \% \mathrm{CNF} / \mathrm{SF}$ fibers showing a crystallinity index of $58.6 \%$. This value is calculated from the IR absorption peaks in the Amide III band of SF, and it indicates the 
ratio of the crystalline regions to the amorphous regions of the silk. Thus, the addition of CNFs does not alter the value of the crystallinity index of the composite fibers if it has no effect on the SF. In other words, the decrease in the crystallinity index of the SF indicates that the CNFs restrict the crystallization of the SF. The SF molecules adopt the random coil conformation in formic acid solution, and $\beta$-sheet crystallization occurs in the SF fibers when formic acid is eliminated from the silk solution (Um et al., 2004) and SF is coagulated in methanol. It seems that the presence of CNFs restricts the crystallization of the SF when formic acid is removed, and methanol penetrates the coagulating dope solution during the coagulation process. $\beta$-sheet crystallization of the SF is a result of the formation of hydrogen bonds between the SF molecules originating from the many polar groups of the $\mathrm{SF}$, including $\mathrm{O}-\mathrm{H}, \mathrm{C}=\mathrm{O}$, and $\mathrm{N}-\mathrm{H}$ functional groups. $\mathrm{CNF}$ also has many $\mathrm{OH}$ groups in the cellulose chain, and hydrogen bonds may be formed between CNFs and the SF. This bonding could prevent hydrogen bonding between the SF molecules, resulting in a decrease in the crystallization of the SF/cellulose blend film (Freddi et al. 1995) and suggesting the occurrence of intermolecular interactions between fibroin and cellulose through hydrogen bonding, as evidenced from the IR spectra.

\section{Morphology of wet-spun filament}

(Figs. 6 and 7) display the surface morphology of the $\mathrm{CNF} / \mathrm{SF}$ composite fibers with varying CNF contents. At a low magnification (x500, Fig. 6), the composite fiber showed stripes in the fiber axis direction, with a relatively smooth surface. Additionally, at high magnification (x10,000, Fig. 7), the regenerated SF fibers (Fig. 7a) showed smooth surfaces. However, rugged surfaces of the CNF/SF composite fibers ( $1-5 \% \mathrm{CNF}$ ) became visible. In particular, the rugged surfaces were more prevalent when a higher amount of CNFs was present. It seems that the rugged surface might be related to CNF aggregates, considering the fiber thickness is on the order of several hundred nanometers. This rugged surface may also be evidence for the presence of CNF aggregates in the CNF/ SF composite.

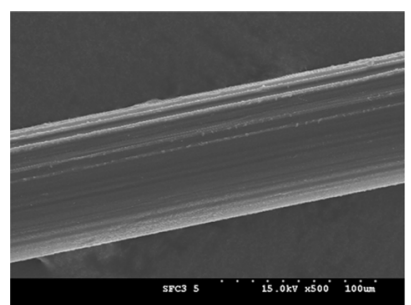

(a)

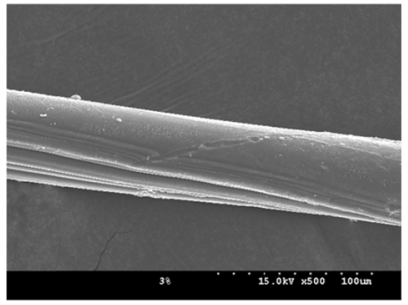

(c)

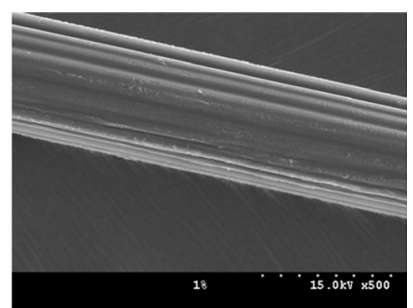

(b)

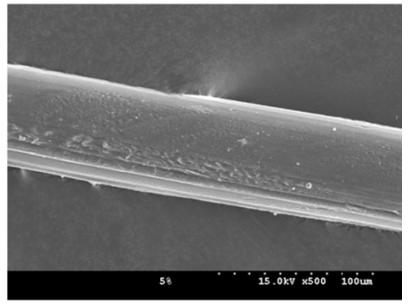

(d)
Fig. 6. SEM images of CNF/regenerated SF filament with various CNF contents at low magnification (x 500): (a) $0 \%$, (b) $1 \%$, (c) $3 \%$, and (d) $5 \%$.

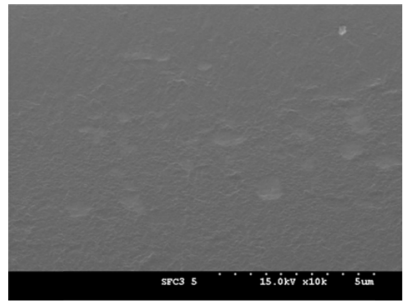

(a)

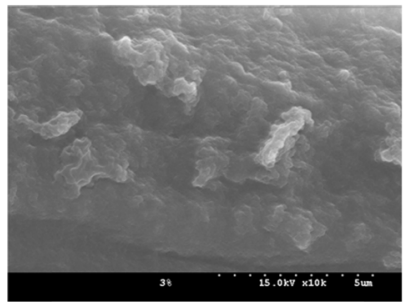

(c)

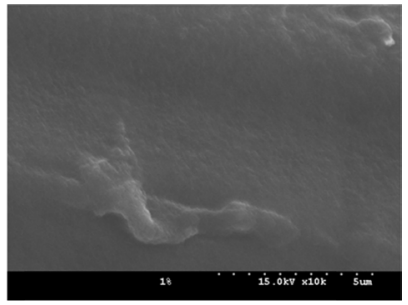

(b)

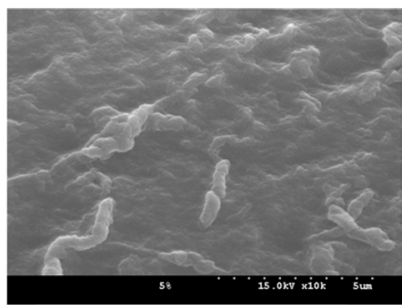

(d)
Fig. 7. SEM images of $\mathrm{CNF} /$ regenerated $\mathrm{SF}$ filament with various CNF contents at high magnification (x 10,000): (a) $0 \%$, (b) $1 \%$, (c) $3 \%$, and (d) $5 \%$.

\section{Acknowledgement}

This study was supported by the Basic Science Research Program through the National Research Foundation of Korea (NRF) funded by the Ministry of Education, Science and Technology (2012042016). 


\section{References}

Aksoy EA, Akata B, Bac N, Hasirci N (2007) Preparation and characterization of zeolite eta-polyurethane composite membranes. J Appl Polym Sci 104, 3378-3387.

Bhat NW, Nadiger GS (1980) Crystallinity in silk fibers: Partial acid hydrolysis and related studies. J Appl Polym Sci 25, 921-932.

Bhatnagar A, Sain M (2005) Processing of cellulose nanofiber reinforced composites. J Reinf Plast Compos 24, 1259-1268.

Brown EE, Laborie MPG (2007) Bioengineering bacterial cellulose/ poly(ethylene oxide) nanocomposites. Biomacromolecules 8 , 3074-3081.

Cho HJ, Um IC (2010) The effect of dissolution condition on the yield, molecular weight, and wet- and electro-spinnability of regenerated silk fibroins prepared by $\mathrm{LiBr}$ aqueous solution. Int $\mathrm{J}$ Indust Entomol 20, 99-105.

Cho HJ, Yoo HJ, Kim JW, Park YH, Bae DG, Um IC (2012) Effect of molecular weight and storage time on the wet- and electrospinning of regenerated silk fibroin. Polym Degrad Stab 97, 10601066.

Chung DE, Um IC (2011) The relationship between the rheological properites and wet spinnability of regenerated silk fibroin solution with various molecular weights and concentrations, 6th International Workshop for East Asian Young Rheologists, Yamagata, Japan, p 63.

Dufresne A, Vignon MR (1998) Improvement of starch film performances using cellulose microfibrils. Macromolecules 31, 26932696.

Freddi G, Romano M, Massafra MR, Tsukada M (1995) Silk fibroin/ cellulose blend films-preparation, structure, and physical-properties. J Appl Polym Sci 56, 1537-1545

Huang J, Liu L, Yao JM (2011) Electrospinning of bombyx mori silk fibroin nanofiber mats reinforced by cellulose nanowhiskers 12 , 1002-1006.

Ki CS, Kim JW, Oh HJ, Lee KH, Park YH (2007) The effect of residual silk sericin on the structure and mechanical property of regenerated silk filament.Int J Biol Macromol 41, 346-353.

Kim HJ, Um IC (2011) The effect of sericin content on the rheological properties and wet spinnability of regenerated silk fibroin solution, 6th International Workshop for East Asian Young Rheologists, Yamagata, Japan, p 66.

Kim HJ, Chung DE, Um IC (2013) Effect of processing conditions on the homogeneity of partially degummed silk evaluated by FTIR spectroscopy. Int J Indust Entomol 25, 54-60.

Klemm D, Kramer F, Moritz S, Lindstrom T, Ankerfors M, Gray D et al. (2011) Nanocelluloses: a new family of nature-based materials. Angew Chem Int Edit 50, 5438-5466.

Ko JS, Um IC (2009) The effect of HPMC concentration on the morphology and post drawing of wet spun regenerated SF/HPMC blend filaments. Int J Indust Entomol 19, 181-185.

Ko JS, Yoon K, Ki CS, Kim HJ, Bae DG, Lee KH et al. (2013) Effect of degumming condition on the solution properties and electrospinnablity of regenerated silk solution. Int J Biol Macromol 55, 161-168.

Li RJ, Zhang YH, Zhu LJ, Yao JM (2012) Fabrication and characterization of silk fibroin/poly(ethylene glycol)/cellulose nanowhisker composite films. J Appl Polym Sci 124, 2080-2086.

Liivak O, Blye A, Shah N, Jelinski LW (1998) A microfabricated wet-spinning apparatus to spin fibers of silk proteins. Structureproperty correlations. Macromolecules 31, 2947-2951.

Meinel L, Hofmann S, Karageorgiou V, Kirker-Head C, McCool J, Gronowicz G et al. (2005) The inflammatory responses to silk films in vitro and in vivo. Biomaterials 26, 147-155.

Minoura N, Aiba S, Gotoh Y, Tsukada M, Imai Y (1995) Attachment and growth of cultured fibroblast cells on silk protein matrixes. J Biomed Mater Res 29, 1215-1221.

Nordqvist D, Idermark J, Hedenqvist MS (2007) Enhancement of the wet properties of transparent chitosan-acetic-acid salt films using microfibrillated cellulose. Biomacromolecules 8, 2398-2403.

Park DJ, Choi Y, Heo S, Cho SY, Jin HJ (2012) Bacterial cellulose nanocrystals-embedded silk nanofibers. J Nanosci Nanotech 12 , 6139-6144.

Phillips DM, Drummy LF, Naik RR, De Long HC, Fox DM, Trulove PC et al. (2005) Regenerated silk fiber wet spinning from anionic liquid solution. J Mater Chem 39, 4206-4208.

Saito T, Nishiyama Y, Putaux JL, Vignon M, Isogai A (2006) Homogeneous suspensions of individualized microfibrils from TEMPOcatalyzed oxidation of native cellulose. Biomacromolecules 7, 1687-1691.

Sakabe H, Ito H, Miyamoto T, Noishiki, Ha WS (1989) In vivo blood compatibility of regenerated silk fibroin. Sen-i Gakkaishi 45, 487490.

Trabbic KA, Yager P (1998) Comparative structural characterization of naturally- and synthetically-spun fibers of Bombyx mori fibroin. Macromolecules 31, 462-471.

Um IC, Kweon HY, Park YH, Hudson S (2001) Structural character- 
istics and properties of the regenerated silk fibroin prepared from formic acid, Int J Biol Macromol 29, 91-97.

Um IC, Ki CS, Kweon H, Lee GK, Ihm DW, Park YH (2004) Wet spinning of silk polymer II. Effect of drawing on the structural characteristics and properties of filament. Int J Biol Macromol 34, 107-119.

Um IC, Kweon HY, Lee KG, Park YH (2003) The role of formic acid in solution stability and crystallization of silk protein polymer. Int J Biol Macromol 33, 203-213.

Um IC, Kweon HY, Park YH (2012) Hemicellulose removal and crystalline structure transition of flax fiber with alkali treatment.
Text Sci Eng 49, 271-278.

Um IC, Kweon HY, Hwang CM, Min BG, Park YH (2002) Structural characteristics and properties of silk fibroin/polyurethane blend films. Int J Indust Entomol 5, 163-170.

Yan JP, Zhou GQ, Knight DP, Shao ZZ, Chen X (2010) Wet-spinning of regenerated silk fiber from aqueous silk fibroin solution: discussion of spinning parameters. Biomacromolecules 11, 1-5.

Yoo YJ, Kim U-J, Um IC (2010) The effect of coagulant and molecular weight on the wet spinnability of regenerated silk fibroin solution. Int J Indust Entomol 21, 145-150. 